\title{
IN VITRO STUDIES ON ENAMEL AND DENTIN ADHESION OF COMPOSITE RESINS IN PATIENTS WITH CHRONIC KIDNEY FAILURE AND HEALTHY PATIENTS
}

\author{
GEORGIANA FLORENTINA GHEORGHE ${ }^{a}$, OANA ELENA AMZA ${ }^{a}$, \\ IOANA SUCIUa, BOGDAN DIMITRIUa, LILIANA GARNEATA ${ }^{b}$, \\ ANDREA MARIA CHISNOIUc, ${ }^{*}$, DOINA PRODAN ${ }^{d}$, \\ MIHAELA PĂSTRAV ${ }^{\mathrm{c}, *}$, SANDA ILEANA CIMPEAN ${ }^{\mathrm{c}, *}$, \\ RADU MARCEL CHISNOIUc
}

\begin{abstract}
The objective of this study was to compare the structure, morphology and enamel-composite and dentin-composite interface in the teeth of patients with chronic renal failure, predialysis and healthy patients, using two methods: atomic force microscopy (AFM) and scanning electron microscopy (SEM). Most of the tested enamel specimens showed a mixed type of failure. The AFM examination indicated a deep engraving for enamel and dentin in sick people compared to healthy people. The average value of the roughness $(\mathrm{Ra})$ in enamel and dentin from healthy patients were significantly different $(p<0.001)$ than from enamel and dentin in patients with renal failure. Regarding the SEM images, the dentin-composite material interface in teeth extracted from healthy patients, has uniformity, compared to the dentin-composite interface in teeth extracted from predialysis patients where a discontinuous hybrid layer with some gaps was identified.
\end{abstract}

Keywords: atomic force microscopy, scanning electron microscopy, enamelcomposite interface, dentin-composite interface, predialysis.

a Carol Davila University of Medicine and Pharmacy, Faculty of Dentistry, 17-23 Calea Plevnei, Bucharest, Romania.

b Carol Davila University of Medicine and Pharmacy, 8 B-dul Eroilor Sanitari, Bucharest, Romania.

c Iuliu Hatieganu University, Faculty of Dental Medicine, 8 Babes str., RO-400012, Cluj-Napoca, Romania.

d Babes-Bolyai University, Raluca Ripan Institute for Research in Chemistry, 31 Fantanele str., RO-400294, Cluj-Napoca, Romania.

* Corresponding author: maria.chisnoiu@umfcluj.ro.

$\ddagger$ Contributed equally to the study with the first author and can be considered main author. 


\section{INTRODUCTION}

The chronic renal failure is a condition whose incidence is constantly increasing, the consequence being the presentation of a large number of patients requesting dental treatments. Moreover, the orodental status considerably influences the evolution and prognosis of the underlying condition. For a correct dental treatment of this category of patients, the dentist must know and understand the systemic and oral manifestations that this condition has and implicitly its treatment.

The alterations in the hard tissue mineralisation of patients with chronic kidney disease are well known today. They are caused by disorders in the metabolism of calcium and phosphorus, increased parathyroid activity and abnormal metabolism of vitamin D [1]. The bone-like changes in dentin were not recognized until 1983, when a study showed the existence of a thinner layer of predentin in the teeth of patients with chronic kidney disease [2]. Moreover, dental changes such as hypoplasia of the enamel of temporary but also permanent teeth, narrowing or calcification of the pulp chamber have been reported $[3,4]$.

It is evident from the literature that the atomic force microscopy (AFM) and scanning microscopy (SEM) are commonly used methods for the analysis of dental structures $[12,16]$. They are especially useful in the studies of the dentin collagen network and changes caused by different chemical agents on the dentin [17]. The changes in the intertubular and peritubular dentin caused by interaction with phosphoric acid, adhesion, structure and other changes in the restoration process can be investigated [13]. It can also be seen the interaction of dentinal adhesives with the hard tissues of the tooth. AFM has been applied in ultramorphological investigations of superficial and deep dentin and its mechanical properties or the identification of the functional width of the enamel-dentin junction $[14,15]$. This method also provides information about the roughness of dentin, enamel and restorative materials, which we tried to highlight in this study $[16,17]$.

Taking into account the previous information but also the fact that modern aesthetic restorative dentistry is completely dependent on the quality and performance of adhesion of dental materials to the dental substrate, there is the possibility of changes in the teeth of patients with chronic renal failure.

The aim of the current study was to compare the structure, morphology and enamel-composite and dentin-composite interface in the teeth of patients with chronic renal failure, predialysis and healthy patients, using two methods: atomic force microscopy (AFM) and scanning electron microscopy (SEM). 


\section{RESULTS AND DISCUSSION}

The observed morphological characteristics of the teeth are described qualitatively and quantitatively. For the quantitative assessment, imaging data and software package available for image analysis were used. In order to meet the statistical nature of the quantitative analysis, images were collected from a variety of different locations (enamel, dentin, composite, and the composite/dentin, enamel/composite interface) on the tooth specimens. Several samples of each type, collected from different patients, were also used. Below are the images obtained and the scanning parameters for each image and the roughness analysis (Figures 1-12).
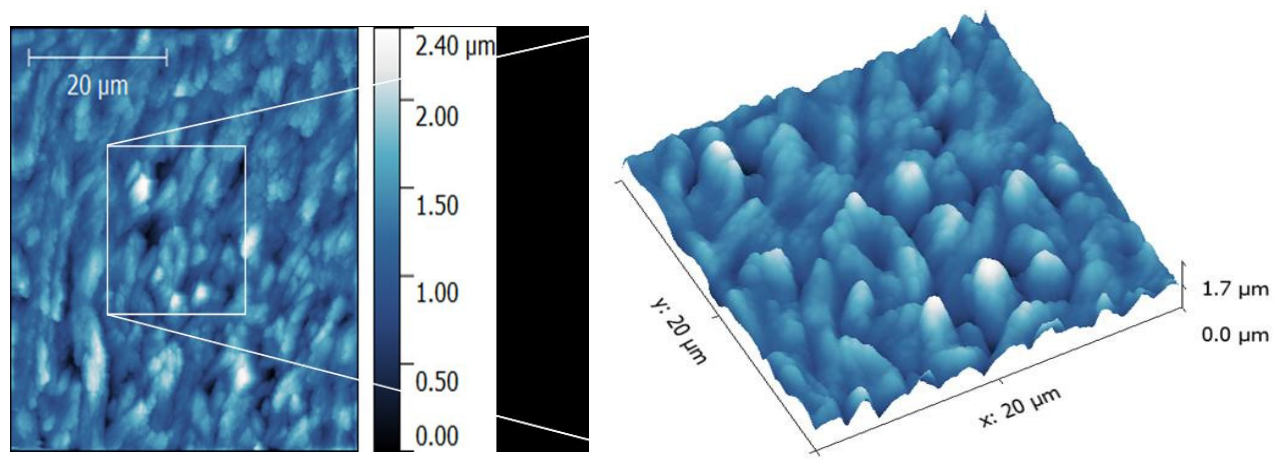

Figure 1. Bidimensional and tridimensional AFM image of the enamel on a healthy patient.

Table 1. Roughness parameters for scanned areas showed in Figure 2

\begin{tabular}{|c|c|c|c|c|c|}
\hline Enamel & $\begin{array}{c}\text { Av } \\
{[\mu \mathrm{m}]}\end{array}$ & $\begin{array}{c}\text { Ra(Sa) } \\
{[\mathbf{n m}]}\end{array}$ & $\begin{array}{c}\text { RMS (Sq) } \\
{[\mathbf{n m}]}\end{array}$ & Sk & Sku \\
\hline $20 \times 20 \mu \mathrm{m}^{2}$ & 0.91 & $161(\underline{+2.26)})$ & 210 & 0.00574 & 0.778 \\
\hline $50 \times 50 \mu \mathrm{m}^{2}$ & 1.202 & $241( \pm 4.67)$ & 307 & 0.0118 & 0.367 \\
\hline
\end{tabular}


The analysis of the surfaces presented in Figures 1-9 leads to the data presented in Tables 1, 2, 3, 4, 5 and $\mathbf{6}$ where the parameters presented refer to:

- Av - mean of heights;

- $\mathrm{Ra}(\mathrm{Sa})$ arithmetic mean roughness;

- $\mathrm{RMS}(\mathrm{Sq})$ mean square deviation (standard deviation) calculated by the formula

$$
S_{q}=\sqrt{\frac{1}{M N} \sum_{k=0}^{M-1} \sum_{l=0}^{N-1}\left[z\left(x_{k}, y_{l}\right)\right]^{2}}
$$

A positive correlation was registered for the parameter Ra and RMS, for which higher values indicate a greater variation of the surface topography.

- $\mathrm{S}_{\mathrm{sk}}$ asymmetry of the height distribution histogram (skewness) calculated by the formula

$$
S_{s k}=\frac{1}{M N \cdot S_{q}^{3}} \sum_{k=0}^{M-1} \sum_{l=0}^{N-1}\left[z\left(x_{k}, y_{l}\right)\right]^{3}
$$

For $S_{s k}=0$, the height distribution is symmetric (Gaussian distribution, totally random variations). The positive asymmetry, Ssk $>0$, indicates a smooth surface with peaks above average, while the negative values Ssk < 0 indicate the presence of many below average heights (pits or depressions in the surface).

- $\quad \mathrm{S}_{\mathrm{ku}}$ flattening the distribution curve of the height values (kurtosis) calculated by the formula

$$
S_{k u}=\frac{1}{M N \cdot S_{q}^{4}} \sum_{k=0}^{M-1} \sum_{l=0}^{N-1}\left[z\left(x_{k}, y_{l}\right)\right]^{4}
$$

reflects the number of extreme heights, 'peakedness' of the surface topography and is a measure of the degree of flattening or vaulting of the distribution curve.

Value $S_{k u}=0$ shows a normal Gaussian distribution, which is a Mesokurtic distribution. An area with a small number of extreme heights (several very high peaks or very deep valleys) will have a large $S_{k u}$, as opposed to an area with mostly moderate heights, which will generate small or negative $S_{k u}$. 
IN VITRO STUDIES ON ENAMEL AND DENTIN ADHESION OF COMPOSITE RESINS...

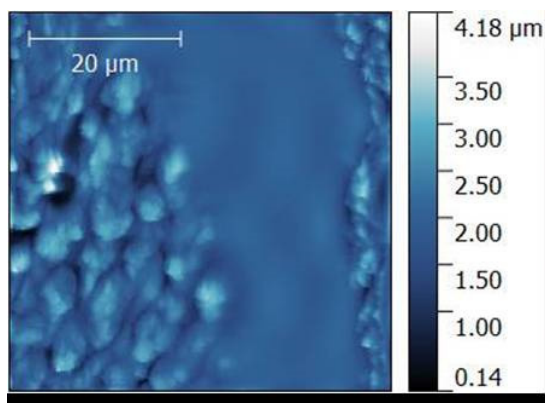

a

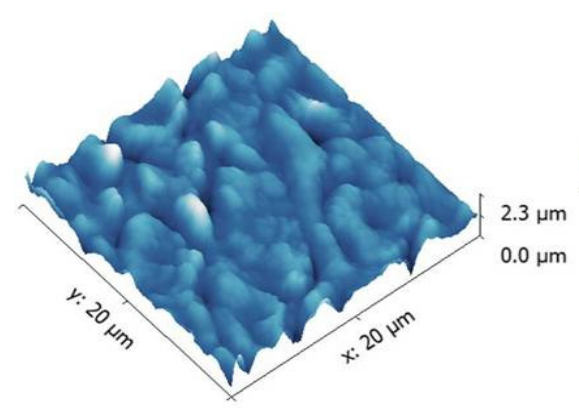

C

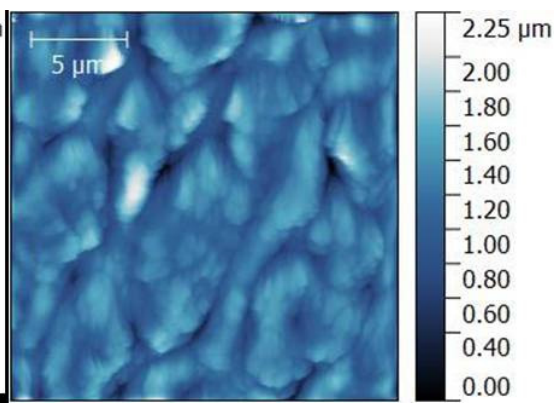

b

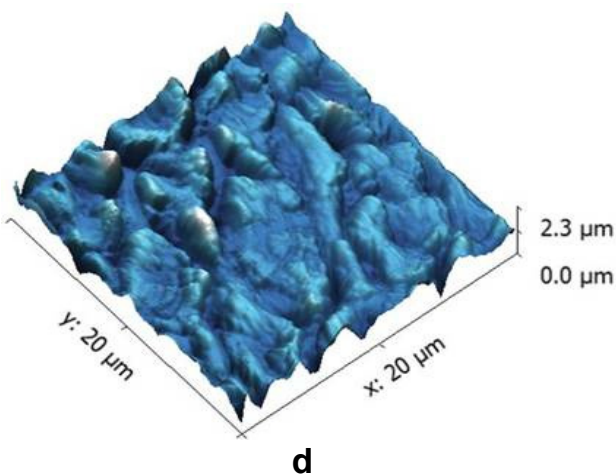

d

Figure 2. AFM images: a,b- bidimensional; c,d - tridimensional of the enamel from the enamel-composite area for extracted teeth in healthy patients.

Table 2. Roughness parameters for the scanned areas showed in Figure 3

\begin{tabular}{|c|c|c|c|c|c|}
\hline & $\begin{array}{c}\text { Av } \\
{[\mu \mathrm{m}]}\end{array}$ & $\begin{array}{c}\text { Ra(Sa) } \\
{[\mathbf{n m}]}\end{array}$ & $\begin{array}{c}\text { RMS (Sq) } \\
{[\mathbf{n m}]}\end{array}$ & Sk & Sku \\
\hline Enamel & & & & & \\
\hline $20 \times 20 \mu \mathrm{m}^{2}$ & 1.177 & $195( \pm 3.56)$ & 252 & -0.197 & 0.824 \\
\hline $50 \times 50 \mu \mathrm{m}^{2}$ & 1.134 & $212( \pm 4.76)$ & 269 & -0.26 & 0.212 \\
\hline Composite & & & & & \\
\hline $50 \times 50 \mu \mathrm{m}^{2}$ & 0.776 & $140( \pm 2.15)$ & 180 & -0.129 & 0.571 \\
\hline $20 \times 20 \mu \mathrm{m}^{2}$ & 1.295 & $112( \pm 5,12)$ & 147 & -0.414 & 1.43 \\
\hline
\end{tabular}




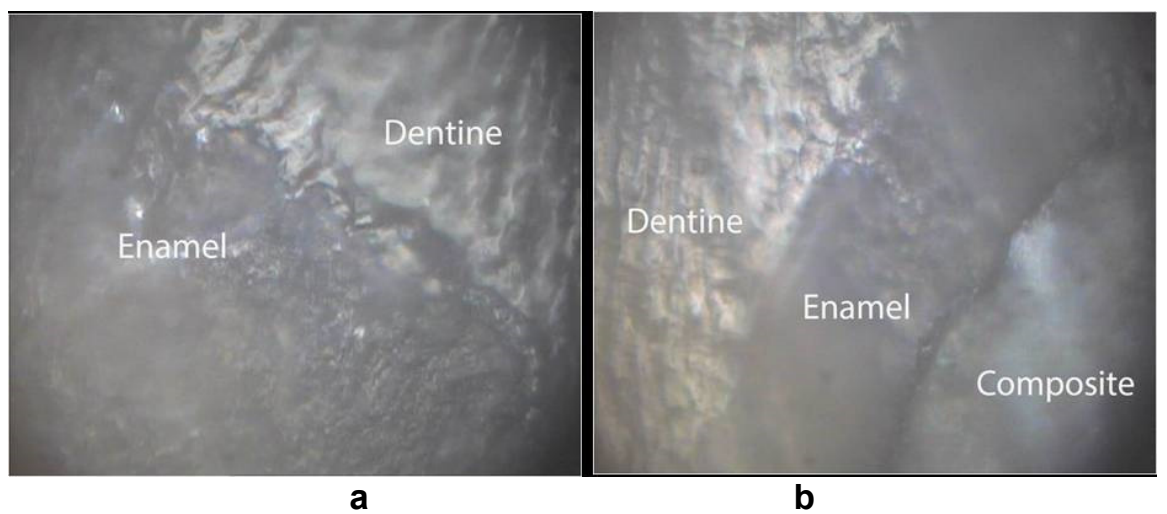

Figure 3. Images of the contact areas: a - enamel-dentin; b - enamel-dentincomposite for extracted teeth in healthy patients
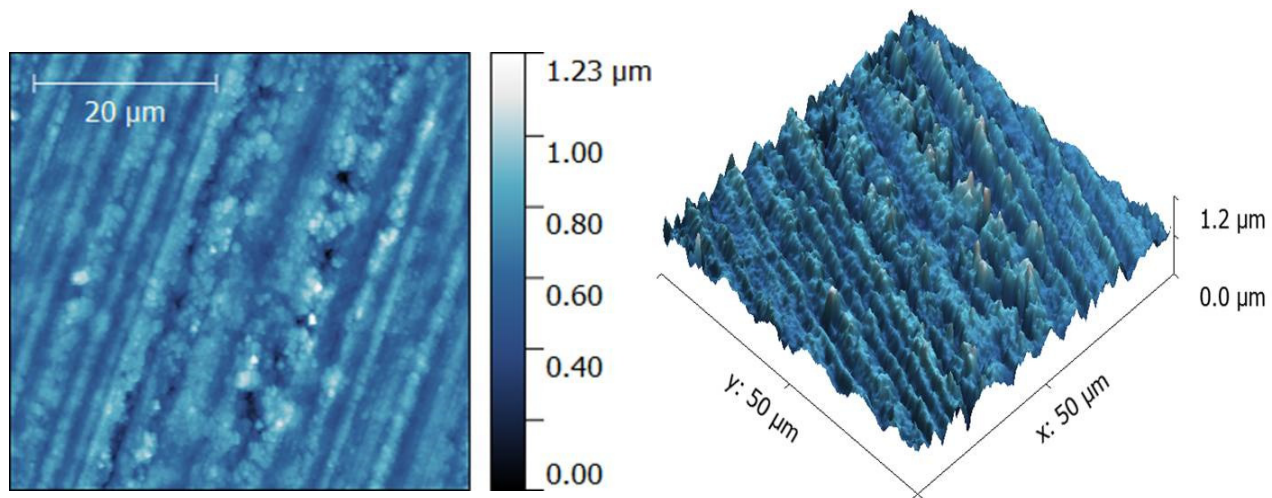

Figure 4. Bidimensional and tridimensional AFM images for Tetric EvoCeram Bulk Fill composite used in this study.

Table 3. Roughness parameters for the scanned areas showed in Figure 4

\begin{tabular}{|c|c|c|c|c|c|}
\hline Composite & $\begin{array}{c}\text { Av } \\
{[\mu \mathrm{m}]}\end{array}$ & $\begin{array}{c}\text { Ra(Sa) } \\
{[\mathrm{nm}]}\end{array}$ & $\begin{array}{c}\text { RMS (Sq) } \\
{[\mathrm{nm}]}\end{array}$ & Sk & Sku \\
\hline $50 \times 50 \mu \mathrm{m}^{2}$ & 0.6733 & $95.5( \pm 3,45)$ & 122 & -0.231 & 0.654 \\
\hline $20 \times 20 \mathrm{a} \mathrm{\mu \textrm {m } ^ { 2 }}$ & 0.3269 & $68.2( \pm 2,13)$ & 88.2 & 0.081 & 0.284 \\
\hline
\end{tabular}




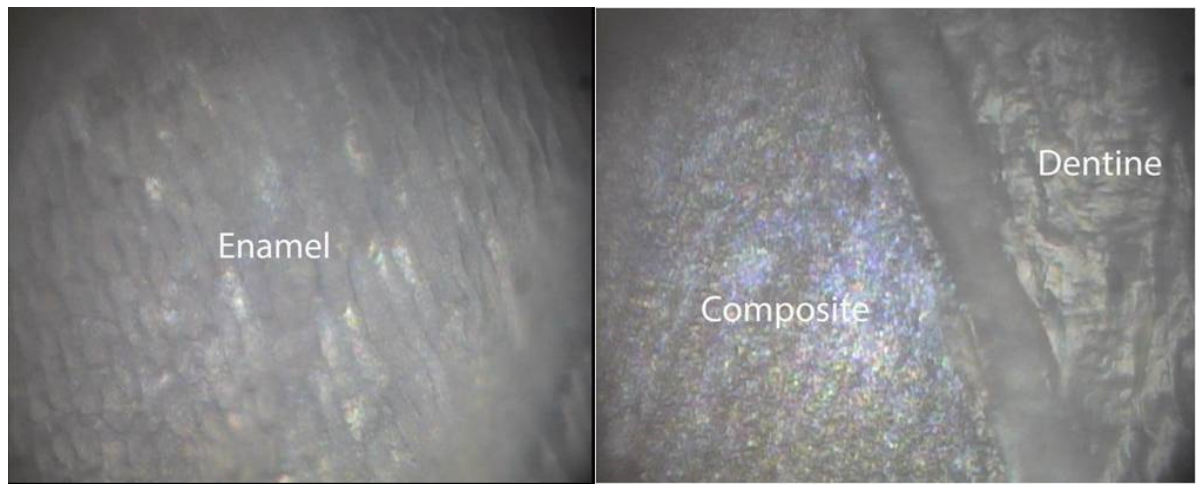

Figure 5. Enamel and composite-dentin interface images, registered on extracted teeth from predialysis patients

Comparing the images in Figures $\mathbf{1}$ and $\mathbf{6}$ it is observed that the surface of the healthy tooth is much more uniform and significantly homogeneous. The AFM images show us similar morphological features, clearly emphasizing that the tooth enamel was not damaged during microscopy recordings.

Roughness is a very important surface property for technological applications, being used for quantitative analysis of changes that the surface may suffer; for example, surface erosion will naturally lead to an increase in its roughness.

Following the assessment of tooth enamel from healthy patients, we found that the surface is irregular, with an average height of $190 \mathrm{~nm}$. The arithmetic roughness of $161 \mathrm{~nm}$ and the mean square deviation of $210 \mathrm{~nm}$ are large values indicating a rough surface. The value 0.00574 of the parameter Ssk indicates an almost symmetrical distribution (Figure 1a), a surface with generally random variations in height, and the value Sku 0.778 also indicates the presence on this surface of several lower heights (Figure 1 b).

The images for tooth enamel from predialysis patients also show irregular surfaces. The Ra value of $178 \mathrm{~nm}$ and the average square roughness $\mathrm{Sq} 231 \mathrm{~nm}$ indicate a rougher surface than the enamel from healthy teeth. The negative value of -0.273 of the $S_{k}$ parameter indicates a surface with recesses, and Sku 0.54 indicates a lower distribution of height values compared to the enamel from healthy teeth.

The assessment of the morphology and roughness at the enamel/ composite contact surface (Figure 7) for teeth extracted from predialysis patients indicates an irregular surface, and the negative value of the parameter $\mathrm{S}_{\mathrm{k}}$ indicates a surface with recesses for both composite and enamel. 


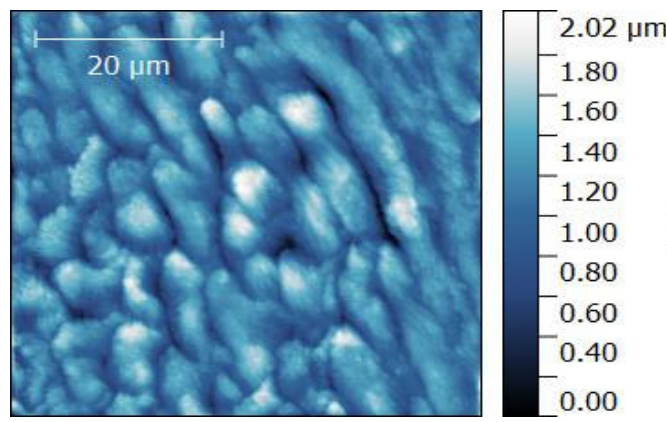

a

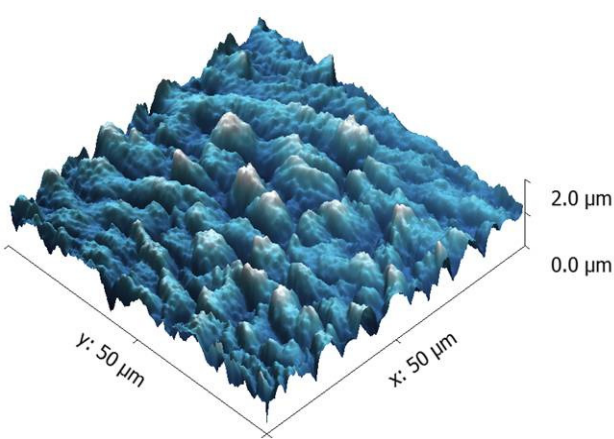

b

Figure 6. AFM images: a- bidimensional; $b$ - tridimensional of the enamel for extracted teeth in predialysis patients.

Tabel 4. Roughness parameters for the scanned areas showed in Figure 6

\begin{tabular}{|c|c|c|c|c|c|}
\hline Enamel & $\begin{array}{c}\text { Av } \\
{[\mu \mathrm{m}]}\end{array}$ & $\begin{array}{c}\text { Ra(Sa) } \\
{[\mathrm{nm}]}\end{array}$ & RMS (Sq) $[\mathrm{nm}]$ & Sk & Sku \\
\hline $20 \times 20 \mu \mathrm{m}^{2}$ & 1.181 & $178( \pm 2.34)$ & 231 & -0.273 & 0.54 \\
\hline $50 \times 50 \mathrm{c} \mu \mathrm{m}^{2}$ & 0.985 & $269( \pm 4.78)$ & 327 & -0.374 & -0.446 \\
\hline
\end{tabular}
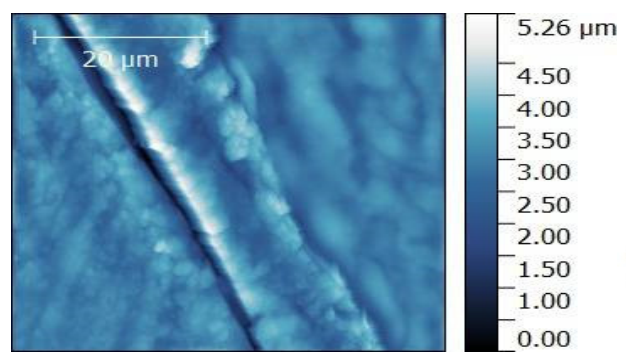

a

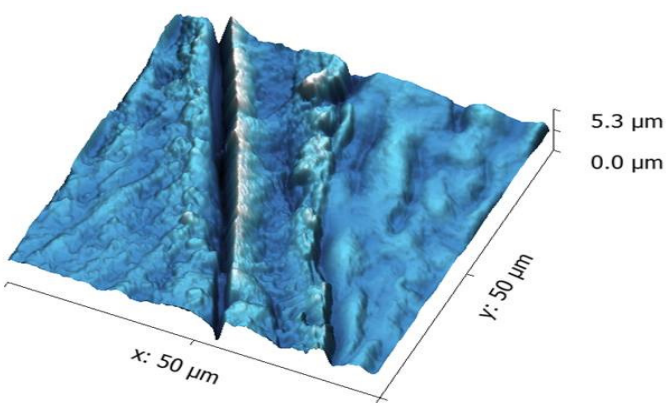

b

Figure 7. AFM images: a- bidimensional; $b$ - tridimensional at the composite -dentin interface for extracted teeth in predialysis patients. 
IN VITRO STUDIES ON ENAMEL AND DENTIN ADHESION OF COMPOSITE RESINS...

Table 5. Roughness parameters for the scanned areas showed in Figure 7

\begin{tabular}{|c|c|c|c|c|c|}
\hline & $\begin{array}{c}\text { Av } \\
{[\mu \mathrm{m}]}\end{array}$ & $\begin{array}{c}\text { Ra(Sa) } \\
{[\mathrm{nm}]}\end{array}$ & RMS (Sq) [nm] & Sk & Sku \\
\hline Dentin & & & & & \\
\hline $20 \times 20 \mu \mathrm{m} 2$ & 0.6657 & $71.7( \pm 4,56)$ & 94.1 & -0.318 & 1.61 \\
\hline Composite & & & & & \\
\hline $50 \times 50 \mu \mathrm{m} 2$ & 1.052 & $176( \pm 3,24)$ & 228 & -0.583 & 1.01 \\
\hline $20 \times 20 \mu \mathrm{m} 2$ & 0.3435 & $56.5( \pm 4,12)$ & 72.0 & -0.39 & 0.466 \\
\hline
\end{tabular}

The arithmetic roughness value of $51.3 \mathrm{~nm}$ at dentin (Figure 8) for teeth from predialysis patients indicates a less rough surface than the composite and enamel from the same samples taken. The high degree of porosity of the dental surfaces is also the most probable reason why the predialysis tooth seems to be fragile and weak; this was frequently noticed during the restoration and recording of both AFM and SEM images. According to these morphological findings, it can be deduced that the influence of treatments on predialysis patients on the teeth is real.

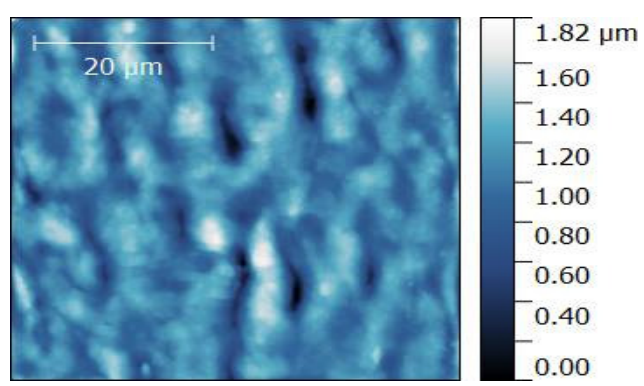

a

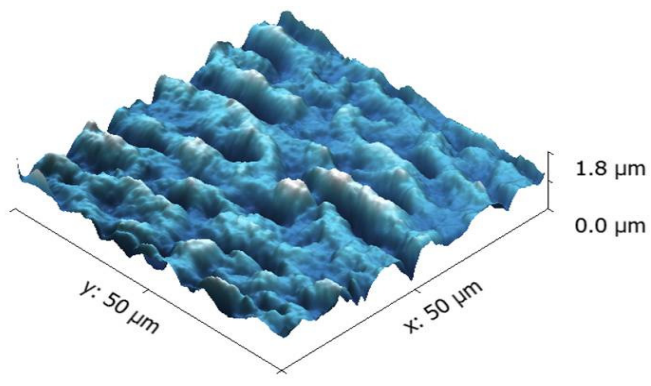

b

Figure 8. AFM images: a- bidimensional; $b$ - tridimensional of dentin from extracted teeth in predialysis patient.

Table 6. Roughness parameters for the scanned areas showed in Figure 9

\begin{tabular}{|c|c|c|c|c|c|}
\hline Dentin & $\begin{array}{c}\text { Av } \\
{[\mu \mathrm{m}]}\end{array}$ & $\begin{array}{c}\mathbf{R a}(\mathbf{S a}) \\
{[\mathrm{nm}]}\end{array}$ & $\begin{array}{c}\text { RMS (Sq) } \\
{[\mathrm{nm}]}\end{array}$ & $\mathbf{S k}$ & Sku \\
\hline $50 \times 50 \mu \mathrm{m}^{2}$ & 1.642 & $177( \pm 2,57)$ & 229 & -0.605 & 1.63 \\
\hline $20 \times 20 \mu \mathrm{m}^{2}$ & 0.7229 & $51.3( \pm 3,45)$ & 67.9 & -0.283 & 4.59 \\
\hline
\end{tabular}


The kidneys play an important role in the endocrine control of calcium and phosphorus metabolism, largely through the formation of vitamin $D_{3}$. During the evolution of the chronic kidney disease, a secondary hyperparathyroidism appears, that is compensating due to the decrease of serum calcium levels in those patients. The main causes of progressive hypocalcemia are: phosphate retention due to decreased renal excretion capacity, decreased production of renal hormone $1,25-(\mathrm{OH})_{2} \mathrm{D}_{3}$ and a reduced skeletal response to the calcium action of parathyroid hormone [5].In the case of chronic renal failure, the decrease in the formation of $1,25-(\mathrm{OH})_{2} \mathrm{D}_{3}$ leads to a decrease in the production of calcium-binding protein in the intestinal mucosa, thus causing a decrease in calcium absorption and initiating the hypocalcemia with increasing serum parathyroid hormone levels [6] .

The mechanism of phosphate retention cannot be separated from alterations in vitamin $\mathrm{D}$ metabolism when considering the pathogenesis of secondary hyperparathyroidism [6].

Most of the enamel specimens tested showed a mixed type of failure, but the bond between the adhesive and the dentin was predominant in several samples. The AFM examination indicates a deep engraving for enamel and dentin in sick people compared to healthy people. The average value of the roughness $(\mathrm{Ra})$ in enamel and dentin from healthy patients were significantly different $(p<0.001)$ than from enamel and dentin in patients with renal failure.

The statistical analysis showed a significant difference between the roughness values obtained for tooth enamel from patients with chronic renal failure and those for tooth enamel of healthy patients. These results could be determined by differences in the micromorphology of demineralised enamel surfaces. The patients with chronic renal failure may have elevated phosphate levels in saliva, as a result of alterations in calcium and phosphorus metabolism. As a result, we can speculate that a high level of phosphates in saliva can lead to the formation of a calcium-phosphate complex that forms a barrier on the tooth surface. Therefore, the diffusion of phosphate ions through the semipermeable enamel via the network of microchannel openings to the external surface can make the enamel more resistant to acid demineralisation, thus causing a low adhesion resistance.

A number of studies have investigated saliva composition and salivary flow in patients with chronic renal failure [7, 8, 9]. Thus, increased salivary concentrations of proteins and minerals were observed, as well as a decrease in salivary flow for both stimulated and unstimulated saliva in patients with chronic kidney disease. The buffer capacity and salivary $\mathrm{pH}$ have been shown to be increased in hemodialysis patients [9].It can be speculated that all these changes recorded at the salivary level may cause increased resistance to enamel demineralisation. 
The results obtained in this study are similar to those presented in the study by Mahmoud et al. [10] on 44 molars collected from healthy patients and hemodialysis patients, to investigate the influence of uremia on the adhesion of composite resins to enamel and dentin, through AFM. Their conclusion was that uremia affects the adhesion of composite resins to enamel and dentin and provides an altered pattern of micromorphology of the demineralised tissues.

Also, in another study, Mahmoud et al. [11] investigated the effect of phosphoric acid concentration and duration of demineralisation on the enamel and dentin of the teeth of hemodialysis patients compared to healthy patients. The authors found that the enamel is much more resistant to acid attack, the higher concentration and longer duration of demineralisation being more effective in patients with chronic renal failure.

The results of the study on the assessment of the composite-enamel and dentin-composite interface by scanning electron microscopy after restoration are highlighted by the SEM images from Figures 9, 11.

The scanning electron microscopy images recorded the characteristics for the adhesive interface and structure in the case of the two lots of extracted teeth studied. Smaller magnifications (40-100x) were initially used for the overall assessment of the restorations and the adhesive interface, then magnifications of 200-1000x for the analysis of the structure of the composite material (C), respectively 1000-1500x for the study of the hybrid layer $(\mathrm{SH})$.
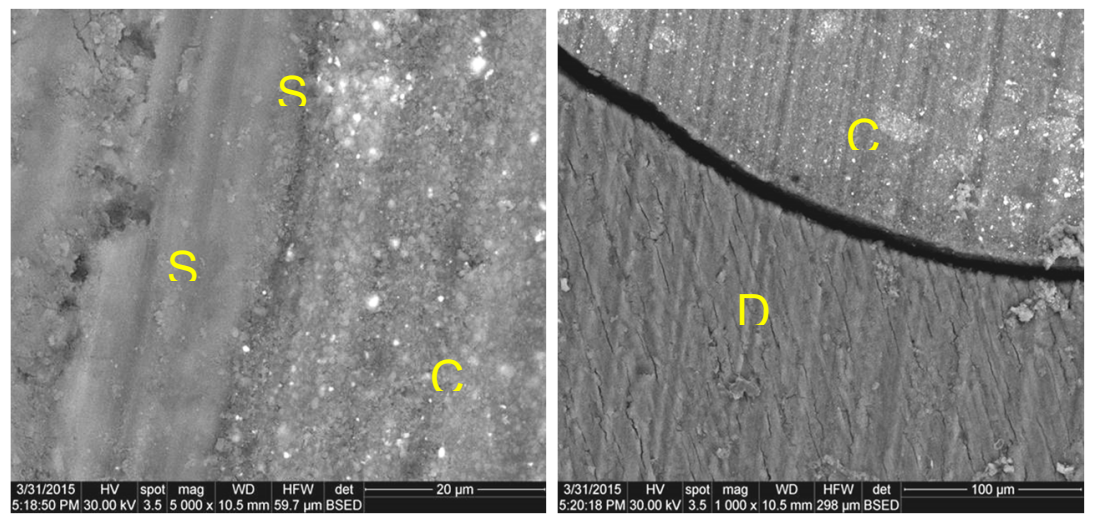

Figure 9. SEM image for extracted teeth from predialysis patients: a: - enamelcomposite interface, magnification of $\times 5000 ; b$ - dentin-composite interface, magnification of $x 1000 ; C$ - composite; D-dentin; SH- hybrid layer; S- enamel 
In the case of samples from lot 1 of teeth extracted from predialysis patients, a continuous interface between composite-adhesive-dental hard tissues was observed, with a thin layer of adhesive, which infiltrated the smear layer (Figure 9.a,b). In several situations, fractures of the dentin were observed in the immediate proximity of the adhesive layer or partial detachment of the adhesive from the dentinal surface (Figure 9). Figures 10 and 12 highlight the elements in the composition of the composite material, enamel and dentin.

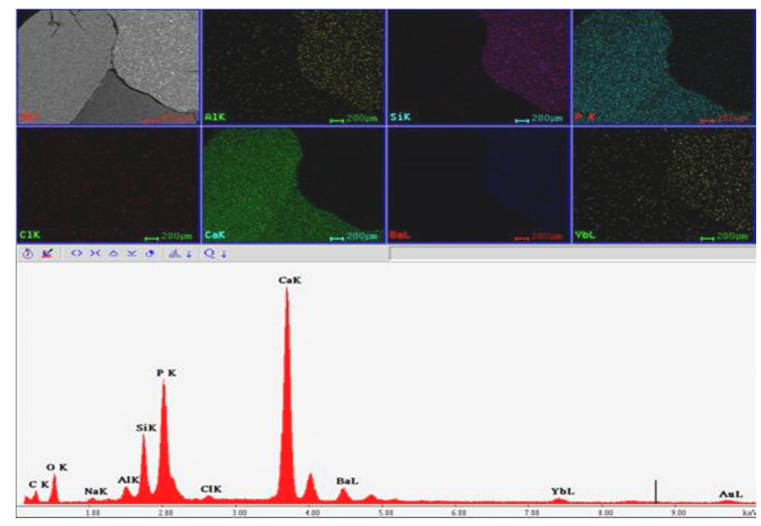

Figure 10. EDAX specters which highlight the elements from the composite-enameldentin interface from extracted teeth in predialysis patients. Coloured image according to the presented elements

The structure of the Tetric EvoCeram Bulk Fill composite material (Figure 11b) presented a hybrid, homogeneous appearance, highlighting 2 types of particles: light in colour, irregular in shape, with diameters varying between 1 and $10 \mu \mathrm{m}$, and dark in colour, with rounded or ovoid shape and diameter between 10-20 $\mu \mathrm{m}$.
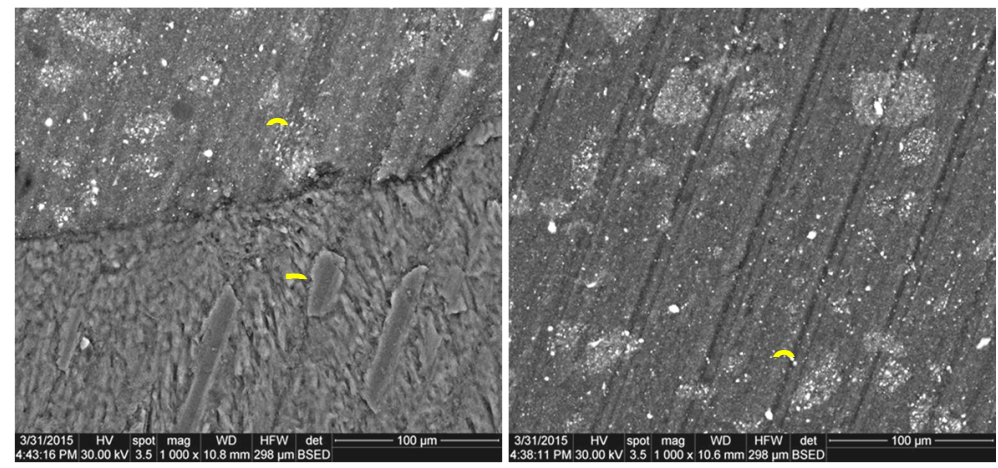

Figure 11. SEM image from extracted teeth in healthy patients: a - dentin-composite interface, magnification of $x 1000$; b- composite material, magnification of $x 1000$;

C- composite; D-dentin; S- enamel 
The dentin-composite material interface on teeth extracted from healthy patients, has a uniformity, compared to the dentin-composite interface in predialysis patients where the formation of a discontinuous hybrid layer was identified. The SEM assessment at the composite-enamel interface for the samples from lot 2 shows a hybrid, well-formed, uniform layer. The morphological changes observed at the enamel-composite interface in the samples from predialysis patients are probably due to the existence of a more porous and fragile dental structure.

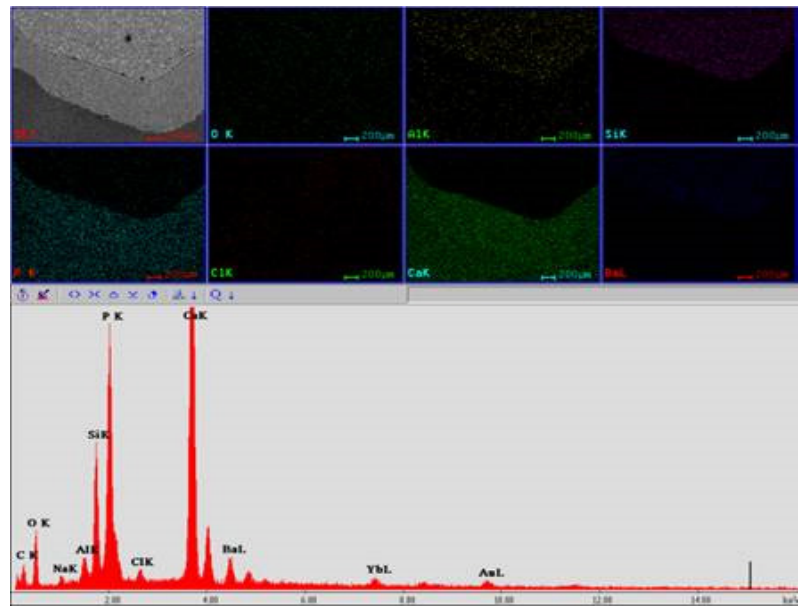

Figure 12. EDAX specters which highlight the elements from the composite-enameldentin interface from extracted teeth in healthy patients Coloured image according to the presented elements

The recent introduction of Bulk Fill restorative materials has rekindled the progressive volume ratio debate, with the introduced composites having a more pronounced polymerization shrinkage compared to the filling volume because the entire mass polymerizes at one time, faster than in small steps. Currently, the Bulk Fill restorative materials are available in different viscosities, low, variable or medium.

A number of studies have investigated the bonding capacity of adhesive systems to either enamel, dentin, or both [18- 24]. Most clinical points of view are for complex prepared cavities and include not only exposed enamel areas and superficial dentin, but also deep dentin areas. The hybrid layer is visualized in SEM images, and is possible only by sectioning the resin/dentin/enamel interfaces, being uniformly formed in the case of teeth from healthy patients.

Gaps and cracks have been observed in the areas of the composite/ dentin interface, indicating that adhesion to dentin is likely to be influenced by several factors than to enamel. The differences observed in these areas may be due to the air drying of the tooth slices and the dehydration of the specimens under vacuum during SEM recordings. However, because these 
gaps or cracks were not only evident in the teeth of predialysis patients, since all samples were treated in the same way, they can be attributed to poorly polymerized hybrid adhesive layers.

The clinical relevance of porosities is unclear: their presence in large numbers at the composite-dental tissue interface can lead to microinfiltrations and failure of restoration by secondary caries or pulpal damage; however, clinical failures due to such porosities are rarely reported. The main purpose of dental adhesives is to ensure retention for composite restorations or cements. In addition to the resistance to mechanical stress, especially to those generated by the stress of the polymerization shrinkage of the composite, a good adhesive must also prevent microinfiltration along the edges of the restoration. Clinical failure of restorations occurs mainly through inadequate sealing rather than loss of retention, resulting in marginal dyschromia.

\section{CONCLUSIONS}

The structure, morphology and enamel-composite-dentin interface on teeth extracted from patients with chronic renal failure, predialysis patients and teeth from healthy patients were compared using two methods: atomic force microscopy (AFM) and scanning electron microscopy (SEM).

The AFM examination indicated a deep engraving for enamel and dentin in predialysis patients compared to healthy patients. The average value of the roughness $(\mathrm{Ra})$ in enamel and dentin from healthy patients were significantly different $(p<0.001)$ than from enamel and dentin in patients with renal failure.

The hybrid layer is visualized in SEM images and is uniformly formed in the case of teeth from healthy patients, while in predialysis patients the formation of a continuous hybrid layer with some gaps was identified.

The morphological changes observed at the enamel-composite interface in the samples from predialysis patients are probably due to the more porous and fragile existing dental structure, probably with influences of alterations in the metabolism of calcium and phosphorus.

The clinical relevance of porosities is unclear: their presence in large numbers at the composite-dental tissue interface can lead to microinfiltrations and failure of restoration by secondary caries or pulpal damage; however, clinical failures due to such porosities are rarely reported.

\section{EXPERIMENTAL SECTION}

To perform this study, we used a total of 40 whole human teeth (molars), free of caries, extracted due to periodontal damage. 
Lot 1 - 20 teeth were obtained from patients with chronic renal failure, predialysis patients, who came to the personal dental office for dental treatment. The patients were sent by the nephrologist from the Nephrology II department of the 'Dr. Carol Davila' Clinical Hospital of Nephrology, Bucharest, where they were monitored for their condition.

Lot 2 - 20 extracted teeth were collected from clinically healthy patients, free of chronic renal failure. All teeth were obtained after signing the patient's informed consent form.

Immediately after extraction, the teeth were washed and subjected to ultrasonic scaling (P5 Newtron, Satelec, Acteon, France) to remove deposits of calculus but also remaining soft tissue, being subsequently stored in physiological saline until use, in order to prevent dehydration.

Cavity preparation protocol

For the application of composite resin restorations, class I cavities were prepared at the level of all teeth with the help of a round bur (Komet, Germany) using light movements and water cooling. After preparation, the cavities were cleaned with physiological saline and the restorations were performed by applying the appropriate adhesive system and then the composite material. The technique used for demineralisation was total etch, with $37 \%$ phosphoric acid in gel form (Total Etch, Ivoclar Vivadent; Schaan, Liechtenstein) for 15 seconds, according to the manufacturer's instructions. The acid was then washed with water for 30 seconds.

The cavities were lightly air dried to avoid the desiccation phenomenon, then the adhesive was applied (Adhese Universal, Ivoclar Vivadent; Schaan, Liechtenstein) according to the manufacturer's recommendations. With the help of an LED photopolymerisation lamp (Bluephase Style, Ivoclar Vivadent; Schaan, Liechtenstein) we photopolymerised the adhesive for 10 seconds.

As a composite material we used a nano-hybrid composite resin (Tetric EvoCeramBulk Fill, Ivoclar Vivadent; Schaan, Liechtenstein; shade IVA) placed in a single layer, molded, photopolymerised for 10 seconds with the same LED lamp and then polished.

After filling all the cavities, the teeth were embedded in transparent self-polymerisable acrylic resin (Duracryl Plus, Spofa Dental, Cehia; shade 0 ) in order to be subjected to further tests.

After embedding the teeth in acrylic resin, all samples were sectioned longitudinally with a Microtom Buehler-IsoMet1000 sectioning machine at 1 $\mathrm{mm}$ intervals. The sectioned teeth were stored in physiological saline before and after the restorative treatment, in order to avoid their dehydration. 


\section{Study of the morphology and roughness of dental surfaces, by atomic force microscopy (AFM)}

The atomic force microscopy (AFM) was used to investigate the morphology and roughness of dental surfaces before and after the restoration of cavities on teeth extracted from healthy patients compared to patients with chronic renal failure, predialysis patients. The surface morphology of the initial samples provides an important reference point for the correct and clear assessment of the effects induced by subsequent treatments.

The images were obtained with a commercial Ntegra Spectra microscope (NT-MDT, Russia), at room temperature, in air, in intermittent contact (semicontact), with rectangular silicon cantilever, with Au reflecting surface (NSG30-A, NT -MDT), nominal elasticity constant $k=40 \mathrm{~N} / \mathrm{m}$, resonance frequency $240-440 \mathrm{kHz}$, peak radius $<10 \mathrm{~nm}$. (NT-MDT).

After acquisition, the images were processed using Nova v1.1.0.1837

For each sample, measurements were performed at three different surfaces for composite, enamel, enamel-composite, dentin-composite contact areas. For the roughness analysis, the statistical parameters calculated for the image on which the 'fit lines' correction was applied were used, through which a second-degree polynomial is found and extracted for each scan line. As the image processing history is important for the validity of the roughness comparison of two or more surfaces - the levelling procedure applied to each image directly affecting the roughness values, as well as the image size for which they are calculated - all AFM data were processed identically: for scan areas, $50 \times 50 \mu \mathrm{m}^{2}, 20 \times 20 \mu \mathrm{m}^{2}$, recorded at a resolution of $256 \times 256$ scan points per image at the same correction (second degree polynomial 'fit lines') applied to each image.

\section{Study of the interface by scanning electron microscopy}

The electron microscopy determinations for tooth samples prepared as for the AFM study, in order to investigate the enamel/composite/dentin interfaces, and the structure of enamel and dentin in healthy patients compared to predialysis patients, were performed using the scanning electron microscope (SEM)QUANTA 133 (FEI Company USA). Several image magnification units were used and recordings were made at the enamel/composite/dentin interface. The tooth samples that were examined by SEM scanning electron microscopy were coated with a conductive layer consisting of gold. The analysis of the chemical composition of the tooth surface was performed using the EDAX method; this is a method of quantifying the elements in the composition of enamel, dentin and composite. 
IN VITRO STUDIES ON ENAMEL AND DENTIN ADHESION OF COMPOSITE RESINS...

\section{REFERENCES}

1. G.P. Wysocki; T.D. Dalley; R.A. Ulan. Oral Surg, Oral Med, Oral Pathol, 1983, 56, 167-173.

2. F. Costantinides; G. Castronovo; E. Vettori; C. Frattini; M.L. Artero; L. Bevilacqua; F. Berton; F. Nicolin; R. Di Lenarda. Int J Dent, 2018, 11, 1-8.

3. S.M. Alamo; C.G. Esteve; G. Sarrion Perez. J Clin Exp Dent, 2011, 3, e112119.

4. C.Y. Yang; Z.F. Chang; Y.P. Chau. Calcif Tissue Int, 2015, 97, 466-475.

5. D.B. Clark; G.P. Wysocki. J Oral Pathol \& Med, 1988, 17, 60-69.

6. J. Bacchetta; J. Bernardor; C. Garnier. Calcif Tissue Int ,2021,108, 116-127.

7. I. Tomás; J.S. Marinho; J. Limeres; M.J. Santos; L. Araújo; P. Diz. Arch oral biol, 2008, 53, 528-532.

8. A.M. Abdellatif; S.A. Hegazy, J.M. Youssef. J Advanced Res,2011, 2, 313-318.

9. C.P. Bots; H.S. Brand; J.H. Poorterman; B.M. Van Amerongen; M. ValentijnBenz; E.C. Veerman. Br Dent J 2007, 202, E7-E7.

10. S.H. Mahmoud; M.A. Sobh; A.R. Zaher; M.H. Ghazy; K.M. Abdel Aziz. J Adhes Dent, 2008, 10, 335-338.

11. S.H. Mahmoud; M.E. Ahmed; K.M. Mahmoud; M.A. Grawish; A.R. Zaher. The $J$ Adhes Dent, 2012, 14, 215-221.

12. F.H. Jones. Surf Sci Rep, 2001, 42, 75-205.

13. G.W. Marshall; M. Balooch; R.J. Tench; J.H. Kinney; S.J. Marshall. Dent Mat, 1993, 9, 265-268.

14. C. Cassinelli; M. Morra. J Biomed Mat Res, 1994, 28, 1427-1431.

15. L. Silaghi-Dumitrescu; A. Mihăilescu; A. Muntean; C. Sarosi; D. Prodan; M. R. Simu; M. Moldovan; A. Kui; M. Pastrav. Studia UBB Chemia. 2019, 1, 44.

16. O.H. Orasan; A.M. Chisnoiu; M.L. Dascălu; O. Păstrav; M. Păstrav; M. Moldovan; R. Chisnoiu. Studia UBB Chemia. 2017, 1, 62.

17. F. El Feninat; S. Elouatik; T.H. Ellis; E. Sacher; I. Stangel. Appl Surf Sci, 2001, 183, 205-215.

18. M. Ferrari; A. Vichi; S. Grandini. Dent Mat, 2001, 17, 422-429.

19. C. Poggio; M. Lombardini; P. Vigorelli; M. Ceci. Scanning, 2013, 35, 366-374.

20. B. Van Meerbeek; J. De Munck; Y. Yoshida; S. Inoue; M. Vargas; P. Vijay; G. Vanherle. Oper Dent-Univ of Washington, 2003, 28, 215-235.

21. M. Toledano; R. Osorio; G. De Leonardi; J.I. Rosales-Leal; L. Ceballos; M. Cabrerizo-Vilchez. Am J of Dent, 2001, 14, 205-210.

22. E.R. Hewlett. J California Dent Assoc 2003, 31, 469-476.

23. L. Brache; M. Özcan. J Adhes Sci and Tech, 2018, 32, 258-271.

24. D. Sucală; C. Sarosi; C. Popa; I. Cojocaru; M. Moldovan; A.G. Mohan. Studia UBB Chemia. 2018, 1, 2. 
